\title{
Lung adenocarcinoma radiosensitivity depends on EGFR signaling pathway or other pathways? -- a research based on gene sequencing and data- independent acquisition (DIA) mass spectrometry
}

Chang Jiang ( $\sim$ jiangch0821@163.com )

First Affiliated Hospital of Bengbu Medical College https://orcid.org/0000-0002-4878-3054

Yi-ting Wang

Shanghai Chest Hospital: Shanghai Jiao Tong University Affiliated Chest Hospital

Xue-ru Zhu

Shanghai Chest Hospital: Shanghai Jiao Tong University Affiliated Chest Hospital

Wen Feng

Shanghai Chest Hospital: Shanghai Jiao Tong University Affiliated Chest Hospital

Wen Yu

Shanghai Chest Hospital: Shanghai Jiao Tong University Affiliated Chest Hospital

Li Sun

First Affiliated Hospital of Bengbu Medical College

Qian Sun

First Affiliated Hospital of Bengbu Medical College

Hao Jiang

First Affiliated Hospital of Bengbu Medical College

Xiao-long Fu

First Affiliated Hospital of Bengbu Medical College https://orcid.org/0000-0001-8127-3884

\section{Research Article}

Keywords: lung adenocarcinoma, inherent radiosensitivity,EGFR,Next-Generation Sequencing,DIA-LC$\mathrm{MS} / \mathrm{MS}$

Posted Date: February 8th, 2021

DOl: https://doi.org/10.21203/rs.3.rs-213072/v1

License: (9) This work is licensed under a Creative Commons Attribution 4.0 International License. Read Full License 


\section{Abstract}

Background: To investigate the biological basis of radiosensitivity difference in lung adenocarcinoma cells, EGFR mutant or a wide variety of signal pathways $₫$

Methods: There were fourteen lung adenocarcinoma cell lines used to carry out experiments in this study. The SF2 experimental test data of fourteen cell lines used in this study are from the previous work we did. EGFR mutation in each cell line detected by Next-Generation Sequencing. Statistical difference in SF2 about fourteen cells used the Kruskal-Wallis test. Correlation between EGFR mutant or not and SF2 analyzed by Student's t-test. The proteomes of the 14 lung adenocarcinoma organisms were identified by DIA in LC-MS/MS proteomic technology. $\mathrm{P}<0.05$ showed statistical significance. $\mathrm{R}$ language software was used to perform the bioinformatics analysis.

Results: The SF2(Surviving fractional 2Gy ) median value is $0.351(0.122-0.825)$. The number of cell lines in the EGFR mutatant-type is five, wild-type is nine. We obtained a mean coverage of 5425 proteins from fourteen lung adenocarcinoma cell lines. There are thirty-nine different expression proteins in total.

Significant differences of SF2 was found among fourteen cell lines $(P=0.004<0.05)$. showed that there is no correlation between the EGFR mutant-type group and the EGFR wild-type group $\mathbb{P}=0.822>0.05 \rrbracket$. The $R$ language analyzed based on proteomic explored that there were thirty-nine DEPs(differently expresse proteins) and four KEGG pathways: ECM-receptor interaction, Focal adhesion, Hematopoietic cell lineage, and Prion diseases. LAMC1, ITGB4/ITGA6, and CD44 participated in multiple signaling pathways. PPI examined that LAMC1, ITGB4/ITGA6, CD44, FLNB, CD59, PRNP interacted more concentratedly.

Conclusion: The inherent radiosensitivity of lung adenocarcinoma cell lines is not determined by the status of EGFR mutant, maybe by other signal pathways such as LAMC1, ITGB4/ITGA6, CD44, and PRNP.

\section{Introduction}

Lung cancer is one of the most common malignant tumors that seriously endanger human health. Radiotherapy is an important modality in the treatment of lung cancer[1]. The conventional fraction pattern and classic split dose used in radiotherapy have remained unitary. There has been a lack of individualized therapeutic regimens based on biological characteristics and the inherent radiosensitivity of tumors. However, the utterly difficult and inconsistent detection of inherent radiosensitivity in patients limits the predictor development and their clinical application[2].

Epidermal growth factor receptor (EGFR) is one of the most common lung adenocarcinoma driver genes and refers to the oncogenes that activate the occurrence, survival, and proliferation [3]. EGFR mutations are found in $47 \%$ of NSCLC patients in the Asia Pacific. Tyrosine kinase inhibitors (TKI) could bring a longer progression-free survival to patients with sensitive mutations [4]. However, it remained unknown if the activation of the EGFR pathway that is the hallmark of EGFR mutation alone is sufficient to indicate radiosensitivity and implement individualized combined radiotherapy.[5]A recent meta-analysis found that overall survival(OS) in the different EGFR mutation groups differed significantly., no difference in 
objective recurrence rate(ORR), however, was demonstrated. In pre-clinical researches of NSCLC, most of the research considered that the EGFR mutant is related to radiosensitivity. Takashi Nakano confirmed that radiosensitivity differences between EGFR mutant-type and wild-type lung cancer cells are larger at lower doses[6]. Bo Xie and colleagues used eight NSCLC cell lines to proved that EGFR gene mutations in non-small-cell lung cancer cells are associated with increased radiosensitivity in vitro [7]. Unfortunately, these pre-clinical researches did not unify the pathological types of lung cancer cell lines.

If the EGFR-mutant which is the hallmark of activating the EGFR signaling pathway in lung adenocarcinoma cannot indicate the response to radiotherapy, which signaling pathway has a chance? Based on genomic or proteomic technology to explore the radiosensitivity basic of lung adenocarcinoma has not been widely used in prior studies [8]. Proteins are the specific executor of life activities. With the development of quantitative Proteomics Technologies Based on Mass, the potential for exploration of proteins which is the undertaker of life function is greater than ever. Mass spectrometry technical, Liquid chromatography coupled with tandem mass spectrometry (LC-MS/MS), has become arguably the most powerful workhorse to measure the different states of biomolecules including protein[9]. Dataindependent acquisition (DIA), one of the digital acquisition schemes of tandem mass spectrometry that is independent of the composition of precursor ions for their fragmentation. DIA data acquisition is not limited by the specified target peptide and can be used for quantitative analysis of unknown proteins and large-scale proteins, which is especially suitable for the study of clinical biomarkers. Bioinformatics analysis by $\mathrm{R}$ language software is generally used to identify key candidate proteins and pathways revealing the differential basic. In summary, by employing quantitative protein mass spectrometry integrated with bioinformatics analysis, we could establish a panel to predict patients' radiosensitivity, further establish a dose adjustment model to adjust treatment regimen and reveal the biological basis of radiosensitivity differences.

Based on the controversy, whether EGFR mutant or not can be used as a stratified indicator of radiosensitivity needs to be assessed in a large number and consistent pathological types of cell lines. In this research, we used fourteen lung adenocarcinoma cell lines, obtained a surviving fraction at 2 Gy(SF2) which is the gold standard for evaluation of radiosensitivity [10], and explored whether exist the inherent difference in radiosensitivity we performed DIA- LC-MS/MS on all lung adenocarcinoma cell lines and then used $\mathrm{R}$ language bioinformatics analysis to obtain differently express proteins (DEPs)and signal pathways.

\section{Materials And Methods}

\section{The SF2 data of lung adenocarcinoma cell lines}

We used fourteen lung adenocarcinoma cell lines, H1975 and HCC827, H1650, H1734, HCC4006, A549, $\mathrm{H} 1437, \mathrm{H} 441, \mathrm{H} 2393, \mathrm{H} 1915, \mathrm{H} 2030, \mathrm{H} 838$. The SF2(surviving fraction at 2Gy)experimental data derived from the previous work we did.[11].Each cells'SF2 value was tested three times in the previous experiment. 


\section{EGFR genomeic sequencing assay}

The nucleic acid extraction operation is carried out according to the operation manual of "Tiangen" nucleic acid extraction. The primers of the EGFR gene were designed by PrimerPremier 5 software. The conditions of PCR amplification were as follows: denaturation at $95{ }^{\circ} \mathrm{C}$ for $5 \mathrm{~min}$, denaturation at $94{ }^{\circ} \mathrm{C}$ for $20 \mathrm{~s}$, annealing at $62{ }^{\circ} \mathrm{C}$ for $30 \mathrm{~s}$ and extension at $52^{\circ} \mathrm{C}$ for $1 \mathrm{~min}$ for $35 \mathrm{cycles}$, and finally extended at $72{ }^{\circ} \mathrm{C}$ for $10 \mathrm{~min}$. The reaction system is $30 \mathrm{uL}(\mathrm{ABI}, 9700)$. (using the BGI2xSuperPCRMix (with dye), BGID2000PlusDNALadder kit of Beijing Liuhe Huada Genome Technology Co., Ltd.) 1\% agarose gel electrophoresis was used to identify the amplified fragments. The electrophoresis results of PCR products were observed by a gel imaging system. The purification of PCR products was carried out according to the standard operating procedure of magnetic beads purification. The purified PCR products were detected by computer detection $(\mathrm{ABI}, 3730 \mathrm{XL})$. Use the phred $\backslash$ phrap software for SNP analysis and export the analysis results.

\section{DIA-LC-MS/MS assay}

Proteomic analysis was performed by data-dependent acquisition (DDA) mass spectrometry assay. Sample preparation and fractionation were performed as previously described [12]. Equal aliquots of the samples in this experiment were pooled into one sample for DDA library generation and quality control. All fractions for DDA library generation were injected into Thermo Scientific Q Exactive HF mass spectrometer connected to an Easy-LLC 1200 chromatography system (Thermo Scientific). Each sample peptides were analyzed in data-independent acquisition (DIA) mode. The DIA cycle contained a full MSselected ion monitoring (SIM) scan, and 30 DIA scans were performed covering a mass range of 350$1650 \mathrm{~m} / \mathrm{z}$ with the following settings: SIM full scan resolution, 60,000 at $200 \mathrm{~m} / \mathrm{z}$; automatic gain control (AGC), 3e6; maximum ion trap (IT) time, 50 ms; profile mode; DIA scan resolution, 30,000; AGC target, 3e6; maximum IT, auto; and normalized collision energy, $30 \mathrm{eV}$. The runtime was $120 \mathrm{~min}$ with a linear gradient of buffer B ( $80 \%$ acetonitrile and $0.1 \%$ formic acid) at a flow rate of $250 \mathrm{~nL} / \mathrm{min}$. Quality control samples (pools of equal aliquots of all the samples in the experiment) were injected in DIA mode at the beginning of the MS study and after every 5 injections throughout the experiment and were used to monitor MS performance. (supplement Table 2)

\section{Statistical analysis}

Each of the fourteen lung adenocarcinoma cells had tested three SF2 data, which was considered as fourteen groups. The data of each group of SF2 were derived from multi-repeated and independent experiments and they were random variables with unknown distribution, which accords with the condition of the Kruskal-Wallis test that hypothetical population distribution does not obey Gaussian distribution, but has the same shape. Therefore, Kruskal-Wallis test was used to test the statistical difference of SF2 among 14 lung adenocarcinomas. Statistical difference between the EGFR mutant-type group and the EGFR wild-type group was assessed using the Student's t-test. All statistical analyses used SPSS V20.0 software (IBM Corporation, Armonk, NY, USA)and GraphPad Prism8.4.3(686), which were conducted in triplicates and repeated at least three times. 


\section{Bioinformatics analysis}

Considered SF2 $=0.3$ as cutoff which is based on literature to classify radiation-sensitive (RS)group cell lines and radiation-resistant(RR) group cell lines[13, 14]. The differently express proteins (DEPs) analysis about RS cell lines were compared with those in RT cell lines with the limma package in R[15]. Gene ontology-biological (GO)process and Kyoto encyclopedia of genes and genomes (KEGG) pathway enrichment analyses were performed using the cluster profile package in $\mathrm{R}[16]$. To consider the potentially biological complexities in which a gene may belong to multiple annotation categories, we developed a "heat plot" to extract the complex association by $\mathrm{R}$ language software[17]. To investigate the molecular basics of these proteins, The online tool STRING (https://string-db.org) was used to analyze the target genes. The selection criterion was the highest confidence (interaction score >0.9). Cytoscape software version 3.2.1 was used to draw the protein-protein interaction (PPI) network.

\section{Results}

\section{Inherent difference exist in lung adenocarcinoma radiosensitivity}

The SF2(Surviving fractional 2Gy) values and mean value distribution of fourteen lung adenocarcinoma cell lines were display in Table1 and Figure1.EGFR mutation type was displayed in Table1. The KruskalWallis test $P$ value $=0.004<0.05$. There exist an inherent difference in lung adenocarcinoma radiosensitivity.

Table 1.SF2 and EGFR mutant type of fourteen lung adenocarcinomas 


\begin{tabular}{|c|c|c|c|}
\hline Cell Line & EGFR muta & tion type & MeanSF2 2 SD \\
\hline H1975 & & 21L858Q+20T790M & $0.45 \pm 0.106$ \\
\hline HCC827 & Mutatnt & 19 del & $0.35 \pm 0.141$ \\
\hline H1650 & & 19 del & $0.5 \pm 0.078$ \\
\hline H1734 & & 19 del & $0.239 \pm 0.092$ \\
\hline HCC4006 & & 19 del & $0.483 \pm 0.076$ \\
\hline A549 & & Wild-type & $0.7 \pm 0.267$ \\
\hline H1437 & & Wild-type & $0.18 \pm 0.283$ \\
\hline $\mathrm{H} 441$ & Wild-type & Wild-type & $0.191 \pm 0.580$ \\
\hline H1563 & & Wild-type & $0.352 \pm 0.045$ \\
\hline $\mathrm{H} 23$ & & Wild-type & $0.122 \pm 0.034$ \\
\hline H1793 & & Wild-type & $0.346 \pm 0.317$ \\
\hline H1915 & & Wild-type & $0.163 \pm 0.177$ \\
\hline H2030 & & Wild-type & $0.825 \pm 0.106$ \\
\hline \multirow[t]{2}{*}{ H838 } & & Wild-type & $0.512 \pm 0.340$ \\
\hline & & & Kruskal-Wallis test Pvalue $=0.004<0.05$ \\
\hline
\end{tabular}

\section{No correlation between EGFR mutation and radiosensitivity}

There are five and nine cell lines with EGFR mutant-type and EGFR wild-type respectively.Students' t-test $P$ value $=0.822>0.05$. There was no significant difference in SF2 between the EGFR mutant-type group and the wild-type group.囚Figure2》

\section{Differential analysis of Radiosensitivity of lung adenocarcinoma DEPs (differently expresse proteins)}

Lung adenocarcinoma cells were divided into sensitive groups and insensitive groups with a 0.3 cut off[13][14]. In total, thirty-nine differential expressed proteins were obtained by defining a foldchange $>1$ and $P$ adjValue $<0.05$ with $R$ language limma package[15] $\otimes$ Figure3a,Figure3b囚. Compared with radiationresistant lung adenocarcinoma cells, radiation-sensitive cells showed an up-regulated expression of 26 proteins and down-regulated expression of 12 proteins(Supplement Table2).The differentially expressed proteins were annotated by GO (gene ontology) by R library GO. [16] GO covered three domains: biological process (BP), cellular component (CC), and molecular function (MF)(Figure4a囚. The four KEGG pathways

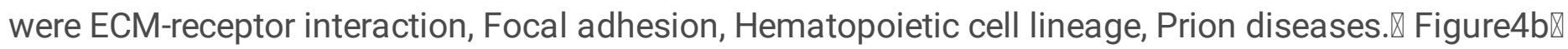

To consider the potentially biological complexities in which a protein may belong to multiple annotation categories and provide information on numeric changes if available, we developed a "heat plot" to extract the complex association by R language software[17]. (Figure5) 
PPI (Protein-protein interaction) for all DEPs used to enrich KEGG (Table6)that can be seen that these proteins can be enriched to the pathway that not only has a direct or indirect correlation between signal pathways but also interact with each other. (Figure6)

\section{Discussion}

NSCLC, as the main category of lung cancer, renders the highest cancer-related mortality in many countries of the world [18]. Radiotherapy is one of the major treatments. However, in the clinical practice of radiotherapy, we have been used single-dose and segmentation mode(58-60Gy,30 times) to carry out NSCLC radiation treatment, resulting in a great difference in clinical efficacy and overall deviation in prognosis. Whether accurate individualized treatment can be determined based on the difference of tumor sensitivity is the necessity of the development of precision medicine. Is there any difference in radiosensitivity among NSCLC patients? We did experiments on fourteen consistent pathological type cell lines.Our results show that there are great differences in SF2 among fourteen lung adenocarcinoma cell lines in vitro(Table 1), and the radiosensitivity of each cell line in this study is close to the results reported in the literature, which reflects the overall reliability of the results of this study. Our observations indicate that inherent radiosensitivity differences exist in lung adenocarcinoma cells obtained from different SF2. But what causes this difference is not clear.

We primarily considered mutations in EGFR, which is a driver that can result in lung adenocarcinoma[18]. EGFR mutations are found in $47 \%$ of NSCLC patients in the Asia Pacific. NCCN guidelines recommended EGFR inhibitor oxitinib for first-line treatment of advanced EGFRm + NSCLC[19]. There is no conclusion on the combination of EGFR inhibitor and radiotherapy. It depends on whether the EGFR mutation affects the outcome of radiotherapy in vivo and whether there is a statistical correlation between the EGFR mutation and the gold standard of radiosensitivity (SF2) in vitro. A recent meta-analysis about the efficacy of definitive chemoradiotherapy in patients with locally advanced unresectable stage III non-small-cell lung cancer found that the presence of EGFR mutations may be indicative of patients overall survival(OS), but no difference in objective recurrence rate(ORR) that was more related to radiosensitivity[5]. This finding is opposed to data previously generated in preclinical studies[6][7][8]. Three articles were using statistical methods analyzed NSCLC cell lines SF2 values and EGFR mutation in the past ten years all have proved that EGFR mutation is related to radiosensitivity. Bo Xie and [8] used eight cell lines, of which three cell lines were repetitive cell-line subtypes in the mutation group. in the study of Takashi Nakano and his colleagues, the pathological types of lung cells were different[6]. The significance of pathological type consistency is that lung squamous carcinoma cell EGFR is not a key driver gene for lung squamous carcinoma, which is another type of NSCLC[20].

In this paper, we considered that there was no significant difference in SF2 between the EGFR mutanttype group and the wild-type group. NSCLC cell lines with EGFR mutation had been first proved to be associated with radiosensitivity in 2006, which is cited as the background of the three articles mentioned above[21]. In the original study, it is that just four cells were observed to have radiosensitivity difference in SF2, which explored 19 cell lines in total[21]. Instead, the effect of radiotherapy resistance was observed 
in the EGFR mutant cell line H1655, which was opposed to most of the mutant cell lines. The author believes that there may be a bypass basic involved in the response of EGFR and radiosensitivity[21]. Recent studies have proved that EGF modulates adhesion by redistributing focal adhesion constituents to adhesion sites. EGF response underlies an EGFR integrin cross-talk under the recruitment of receptor distal events[22]. EGFR might distinguish between radiosensitivity and radiotherapy resistance in a small number of NSCLC cell lines, but in a larger cell sample with a consistent pathological type, EGFR mutation status was insufficient to describe the difference in radiosensitivity. Our results initially raised questions about the use of a single standard of reference, EGFR mutant-or-not, for the diagnosis of radiosensitivity. However, there is no significant correlation between EGFR mutation and radiosensitivity, as expected, cause increasing evidence in cell line models suggests that the real situation may be substantially more complex. Just like organisms as a whole, individual cells can respond to radiation by the whole cell as a system, not as a part. Lung adenocarcinoma cells' difference in radiosensitivity through changes in the activities of biomolecules that integrate signaling pathways and networks.

After the concept of proteomics has been put forward, the technology of proteomics was initially used the electrophoretic gel stained with coomassie brilliant blue, and the staining intensity was quantitatively analyzed. However, limited by staining agents and experimental techniques [23], it has now been replaced by non-gel separation techniques such as liquid chromatography. The principle of liquid chromatographytandem mass spectrometry (Liquid chromatography coupled with tandem mass spectrometry, LCMS/MS) is that protein samples are transformed into moving charged ion fragments, which are separated and identified according to their different mass-charge ratios. Protein and peptide mass spectrometry convert protein molecules into gas-phase ions through ionization sources. Proteins with a specific mass-charge ratio are separated in the electromagnetic field of the mass spectrometer, and unknown proteins are identified. Mass spectrometry can obtain more information, fast analysis speed, high sensitivity, and high resolution. In the further stage of data analysis, DDA and DIA are developed. In this method, the protein samples were digested into peptides and ionized for mass spectrometry analysis. Both DDA and DIA initially need full-scan mass spectra to cleave the signals of peptides that are higher than noise, but DDA is selectively cleaved, and the peptides are matched with the maps of the database to determine the types of proteins. Because most of the cleavage methods of selective random extraction tend to high abundance peptides, the information loss of low abundance peptides is large, and there are high data loss and randomness of collection [24]. DIA data-independent acquisition (Data-independent acquisition, DIA) technology divides the full scanning range into several windows, and then monitors all ions in each window, splits, and extracts all ion information in the sample without omission or difference. DIA technology realizes high data coverage and high reproducibility on this basis, improves the amount of data for a single analysis, reduces the missing and null values of each collection, and improves the reproducibility of identification,quantitative stability and accuracy. The biggest advantage of DIA compared with DDA lies in the efficient determination of extremely low abundance protein molecules in complex samples, which greatly improves the credibility of quantitative analysis and has higher quantitative accuracy and repeatability [25]. Identification of radiosensitive proteins by DIA proteomics has been used in prostate cancer tissue samples, which obtained a mean coverage of 5660 proteins from 
fresh tissue biopsies; with the principal, post-radiation change observed is an increase in levels amongst a total of 49 proteins exhibiting abundance changes.

We used DIA proteomics and bioinformatics analysis to explore differently express proteins (DEPs)and signal pathways, which may reveal the radiosensitivity-specific pathway maps. Our results displayed that there were a total of thirty-nine differential proteins obtained. GO and KEGG showed that signal pathways are ECM-receptor interaction, Focal adhesion, Hematopoietic cell lineage, Prion diseases. Analysis of correspondence between the signal pathway and DEPs combining with the analysis of protein-protein interaction(PPI) showed that the following proteins may play a more important role in the radiosensitivity of lung adenocarcinoma.LAMC1, ITGB4/ITGA6, and CD44 participated in multiple signaling pathways, demonstrated by a centplot. PPI founded that LAMC1, ITGB4/ITGA6, CD44, PRNP interacted more concentratedly. So it is believed that LAMC1, ITGB4/ITGA6, CD44, and PRNP may be the node proteins that weave the signal pathway into a network.(suplement Fig. 2)Among them, ITGB4/ITGA6, CD44 has been proved to be related to radiosensitivity in previous studies, and the details are shown in supplement table 4. The correlation between LAMC1 and PRNP and radiosensitivity has not been reported in previous studies.

In the KEGG signal pathway, the "ECM receptor interaction pathway" and "focal adhesion pathway" have similarities in function. Prion disease pathway and Hematopoietic cell lineage are new signal pathways in the research field of radiosensitivity. (Supplement Table 5)

To describe the radiosensitivity-specific pathway maps should optimize the DEPs integrating into the pathway networks. Differentially expressed proteins-signal pathways correspondence analysis and the PPI analysis showed that proteins, LAMC1, ITGB4/ITGA6, andCD44, not only participate in multiple signal pathways but also interact more closely with each other. Cells participate in the role of radiation as a complex system, and the signal pathways involved should also be a network of signal pathways connected in series with each other[45]. With this in mind, these proteins may be the key proteins that "weave" the network of the radiosensitivity-specific pathway, ECM-receptor interaction, Focal adhesion, Hematopoietic cell lineage, Prion diseases.

The advantages of this study are mainly reflected in three aspects. Firstly, we initially discussed the correlation between the lung adenocarcinoma mutation state of EGFR and radiosensitivity. Given the inconsistency between the results of pre-clinical research and clinical observation, we discussed the reasons for this result, that is, preclinical studies are often based on a small number of cells, and the pathological types are not consistent. Secondly, the SF2 values of fourteen lung adenocarcinoma cell lines were measured in the previous work, and each cell was repeated three times, which is a more reliable result. Therefore, we analyzed the correlation between EGFR mutation and SF2 in the lung adenocarcinoma cell line with EGFR as the driving gene. Thirdly, based on the most advanced proteomics technology, DIA-LC-MS/MS, we used a proteomic analytical method to explore the radiosensitive signal pathways of the lung adenocarcinoma cell lines. The results include not only the signal pathways that have been verified in previous studies but also the signal pathways explored for the first time. However, 
the deficiency of this study is that the results about proteins and signal pathways have not been verified in vivo or in vitro.

\section{Conclusion}

The inherent radiosensitivity of lung adenocarcinoma was determined by the regulation of complex multisignal pathway basics, which can not be described by a single pathway. The key pathways affecting the inherent radiosensitivity of lung adenocarcinoma were found in ECM-receptor interaction, Focal adhesion, Hematopoietic cell lineage, and Prion diseases, not in the EGFR pathway. This finding will be further verified by computational Biology and experiments.

\section{Declarations}

\section{Acknowledgements}

Thank to COBIOER BIOSCIENCE CO.,LTD for providing cell lines,Shanghai Applied Protein Technology CO.,LTD for providing DIA-LC-MS/MS proteomics services, BGI TECH SOLUTIONS (BEIJING LIUHE) COI.,LTD for providing EGFR gene sequencing services.

\section{Authors' contributions}

Chang Jiang,Xiao-long Fu,Xue-ru Zhu conceptualized and designed experiments. Yi-ting Wang performed cell culture. Chang Jiang analyzed and visualized data. Chang Jiang and Xiao-long Fu co-wrote the paper. All authors edited the paper. All authors read and approved the final manuscript.

\section{Funding}

This work was supported by National Natural Science Foundation of China Grant Number 92059206 and Project Fund of Scientific Research and Innovation Program for postgraduates of Bengbu Medical College.

\section{Data availability statement}

The data used in present study are available from corresponding author on request.

\section{Ethics approval and consent to participate}

Not applicable.

\section{Consent for publication}

Not applicable.

\section{Competing interests}


The authors declare that they have no competing interests.

\section{References}

1. Gadgeel SM, Ramalingam SS, Kalemkerian GP. Treatment of lung cancer. Radiol Clin North Am. 2012 Sep;50(5):961 - 74. doi: 10.1016/j.rcl.2012.06.003. PMID: 22974781.

2. Chen M, Liu P, Chen Y, Chen Z, Shen M, Liu X, Li X, Li A, Lin Y, Yang R, Ni W, Zhou X, Zhang L, Tian Y, Li J, Chen J. Long Noncoding RNA FAM201A Mediates the Radiosensitivity of Esophageal Squamous Cell Cancer by Regulating ATM and mTOR Expression via miR-101. Front Genet. 2018 Dec 5;9:611. doi: 10.3389/fgene.2018.00611. PMID: 30574162; PMCID: PMC6292217.

3. Wang S, Shi J, Ye Z, Dong D, Yu D, Zhou M, Liu Y, Gevaert O, Wang K, Zhu Y, Zhou H, Liu Z, Tian J. Predicting EGFR mutation status in lung adenocarcinoma on computed tomography image using deep learning. Eur Respir J. 2019 Mar 28;53(3):1800986. doi: 10.1183/13993003.00986-2018. PMID: 30635290; PMCID: PMC6437603.

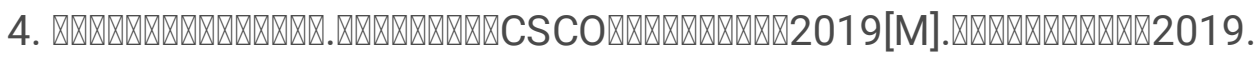

5. Qin Q, Peng B, Li B. The impact of epidermal growth factor receptor mutations on the efficacy of definitive chemoradiotherapy in patients with locally advanced unresectable stage III non-small cell lung cancer: a systematic review and meta-analysis. Expert Rev Anticancer Ther. 2019 Jun;19(6):533-9. doi: 10.1080/14737140.2019.1621754. Epub 2019 May 28. PMID: 31104529.

6. Zhong X, Luo G, Zhou X, Luo W, Wu X, Zhong R, Wang Y, Xu F, Wang J. Rad51 in regulating the radiosensitivity of non-small cell lung cancer with different epidermal growth factor receptor mutation status. Thorac Cancer. 2016 Jan;7(1):50-60. doi: 10.1111/1759-7714.12274. Epub 2015 May 19. PMID: 26816539; PMCID: PMC4718133.

7. Xie B, Sun L, Cheng Y, Zhou J, Zheng J, Zhang W. Epidermal growth factor receptor gene mutations in non-small-cell lung cancer cells are associated with increased radiosensitivity in vitro. Cancer Manag Res. 2018 Sep 13;10:3551-3560. doi: 10.2147/CMAR.S165831. Erratum in: Cancer Manag Res. 2018 Sep 26;10:3983. PMID: 30271203; PMCID: PMC6145635.

8. Yong-ying MU, Pei-ming GU, Bo MA, Wen-xiu YAN. WANG Dao-ping, PAN Ying-hong. Advancements in Quantitative Proteomics Technologies Based on Mass Spectrometry. Biotechnology Bulletin. 2017;33(9):73-84.

9. Zhang F, Ge W, Ruan G, Cai X, Guo T. Data-Independent Acquisition Mass Spectrometry-Based Proteomics and Software Tools: A Glimpse in 2020. Proteomics. 2020 Sep;20(17-18):e1900276. doi: 10.1002/pmic.201900276. Epub 2020 May 19. PMID: 32275110.

10. Matsui T, Nuryadi E, Komatsu S, Hirota Y, Shibata A, Oike T, Nakano T. Robustness of Clonogenic Assays as a Biomarker for Cancer Cell Radiosensitivity. Int J Mol Sci. 2019 Aug 25;20(17):4148. doi: 10.3390/ijms20174148. PMID: 31450688; PMCID: PMC6747107.

11. $X X X X X X X X X X$. 
12. Wiśniewski JR, Zougman A, Nagaraj N, Mann M. Universal sample preparation method for proteome analysis. Nat Methods. 2009 May;6(5):359-62. doi:10.1038/nmeth.1322. Epub 2009 Apr 19. PMID: 19377485.

13. Carmichael J, Degraff WG, Gamson J, Russo D, Gazdar AF, Levitt ML, Minna JD, Mitchell JB. Radiation sensitivity of human lung cancer cell lines. Eur J Cancer Clin Oncol. 1989 Mar;25(3):527 34. doi: 10.1016/0277-5379(89)90266-6. PMID: 2539297.

14. Sirzén F, Zhivotovsky B, Nilsson A, Bergh J, Lewensohn R. Higher spontaneous apoptotic index in small cell compared with non-small cell lung carcinoma cell lines; lack of correlation with Bcl-2/Bax. Lung Cancer. 1998 Oct;22(1):1-13. doi: 10.1016/s0169-5002(98)00066-x. PMID: 9869102.

15. Sand M, Skrygan M, Sand D, Georgas D, Hahn SA, Gambichler T, Altmeyer P, Bechara FG. Expression of microRNAs in basal cell carcinoma. Br J Dermatol. 2012 Oct;167(4):847-55. doi:10.1111/j.13652133.2012.11022.x. Epub 2012 Aug 20. PMID: 22540308.

16. Yu D, Li Y, Ming Z, Wang H, Dong Z, Qiu L, Wang T. Comprehensive circular RNA expression profile in radiation-treated HeLa cells and analysis of radioresistance-related circRNAs. PeerJ. 2018 Jun 15;6:e5011. doi: 10.7717/peerj.5011. PMID: 29922514; PMCID: PMC6005163.

17. Guangchuang Yu L-G, Wang Y, Han, Qing-Yu H. clusterPro-filer: an r package for comparing biological themes among gene clusters.OMICS: A Journal of Integrative Biology, 16(5):284-287, May 2012.

18. Howlader N, Forjaz G, Mooradian MJ, Meza R, Kong CY, Cronin KA, Mariotto AB, Lowy DR, Feuer EJ. The Effect of Advances in Lung-Cancer Treatment on Population Mortality. N Engl J Med. 2020 Aug 13;383(7):640-649. doi: 10.1056/NEJMoa1916623. PMID: 32786189.

19. Soria JC, Ohe Y, Vansteenkiste J,et al. Osimertinib in untreated EGFR-mutated advanced non-smallcell lung cancer[J]. N Engl J Med. 2018;378(2):113-25.

20. Heist RS, Sequist LV, Engelman JA. Genetic changes in squamous cell lung cancer: a review. J Thorac Oncol. 2012 May;7(5):924-33. doi:10.1097/JTO.0b013e31824cc334. PMID: 22722794; PMCID: PMC3404741.

21. Das AK, Sato M, Story MD, Peyton M, Graves R, Redpath S, Girard L, Gazdar AF, Shay JW, Minna JD, Nirodi CS. Non-small-cell lung cancers with kinase domain mutations in the epidermal growth factor receptor are sensitive to ionizing radiation. Cancer Res. 2006 Oct 1;66(19):9601-8. doi:

10.1158/0008-5472.CAN-06-2627. PMID: 17018617.

22. Balanis N, Carlin CR. Mutual cross-talk between fibronectin integrins and the EGF receptor: Molecular basis and biological significance. Cell Logist. 2012 Jan 1;2(1):46-51. doi: 10.4161/cl.20112. PMID: 22645710; PMCID: PMC3355975.

23. Penque D. Two-dimensional gel electrophoresis and mass spectrometry for biomarker discovery. Proteomics Clin Appl. 2009 Feb;3(2):155-72. doi:10.1002/prca.200800025. Epub 2008 Dec 23. PMID: 26238616.

24. Broeckling CD, Hoyes E, Richardson K, Brown JM, Prenni JE. Comprehensive Tandem-MassSpectrometry Coverage of Complex Samples Enabled by Data-Set-Dependent Acquisition. Anal 
Chem. 2018 Jul 3;90(13):8020-8027. doi: 10.1021/acs.analchem.8b00929. Epub 2018 Jun 15. PMID: 29846054.

25. Pappireddi N, Martin L, Wühr M. A Review on Quantitative Multiplexed Proteomics. Chembiochem. 2019 May 15;20(10):1210-1224. doi: 10.1002/cbic.201800650. Epub 2019 Apr 18. PMID: 30609196; PMCID: PMC6520187.

26. Hohenester E, Yurchenco PD. Laminins in basement membrane assembly. Cell Adh Migr. 2013 JanFeb;7(1):56-63. doi:10.4161/cam.21831. Epub 2012 Oct 17. PMID: 23076216; PMCID: PMC3544787.

27. Barderas R, Mendes M, Torres S, Bartolomé RA, López-Lucendo M, Villar-Vázquez R, Peláez-García A, Fuente $\mathrm{E}$, Bonilla F, Casal JI. In-depth characterization of the secretome of colorectal cancer metastatic cells identifies key proteins in cell adhesion, migration, and invasion. Mol Cell Proteomics. 2013 Jun;12(6):1602-20. doi:10.1074/mcp.M112.022848. Epub 2013 Feb 26. PMID: 23443137; PMCID: PMC3675817.

28. Tanaka H, Kawaguchi M, Shoda S, Miyoshi T, Iwasaki R, Hyodo F, Mori T, Hara A, Tomita H, Matsuo M. Nuclear Accumulation of $\beta$-Catenin in Cancer Stem Cell Radioresistance and Stemness in Human Colon Cancer. Anticancer Res. 2019 Dec;39(12):6575-6583. doi: 10.21873/anticanres.13873. PMID: 31810923.

29. Stewart RL, West D, Wang C, Weiss HL, Gal T, Durbin EB, O'Connor W, Chen M, O'Connor KL. Elevated integrin a6 $\beta 4$ expression is associated with venous invasion and decreased overall survival in nonsmall cell lung cancer. Hum Pathol. 2016 Aug;54:174-83. doi: 10.1016/j.humpath.2016.04.003. Epub 2016 Apr 20. PMID: 27107458; PMCID: PMC4938774.

30. Yang L, Zhang X, Hou Q, Huang M, Zhang H, Jiang Z, Yue J, Wu S. Single-cell RNA-seq of esophageal squamous cell carcinoma cell line with fractionated irradiation reveals radioresistant gene expression patterns. BMC Genomics. 2019 Jul 25;20(1):611. doi: 10.1186/s12864-019-5970-0. PMID: $31345182 ;$ PMCID: PMC6659267.

31. Hu T, Zhou R, Zhao Y, Wu G. Integrin a6/Akt/Erk signaling is essential for human breast cancer resistance to radiotherapy. Sci Rep. 2016 Sep 14;6:33376. doi: 10.1038/srep33376. PMID: 27624978; PMCID: PMC5022055.

32. Stewart RL, West D, Wang C, Weiss HL, Gal T, Durbin EB, O'Connor W, Chen M, O'Connor KL. Elevated integrin a6 $\beta 4$ expression is associated with venous invasion and decreased overall survival in nonsmall cell lung cancer. Hum Pathol. 2016 Aug;54:174-83. doi: 10.1016/j.humpath.2016.04.003. Epub 2016 Apr 20. PMID: 27107458; PMCID: PMC4938774.

33. Yang L, Zhang X, Hou Q, Huang M, Zhang H, Jiang Z, Yue J, Wu S. Single-cell RNA-seq of esophageal squamous cell carcinoma cell line with fractionated irradiation reveals radioresistant gene expression patterns. BMC Genomics. 2019 Jul 25;20(1):611. doi: 10.1186/s12864-019-5970-0. PMID: 31345182; PMCID: PMC6659267.

34. Déry MA, Jodoin J, Ursini-Siegel J, et al. Endoplasmic reticulum stress induces PRNP prion protein gene expression in breast cancer[J]. Breast cancer research. 2013;15(2):R22. 
35. Sollazzo V, Galasso M, Volinia S, et al. Prion proteins (PRNP and PRND) are over-expressed in osteosarcoma[J]. J Orthop Res. 2012;30(6):1004-12.

36. Choi J, Yoon YN, Kim N, Park CS, Seol H, Park IC, Kim HA, Noh WC, Kim JS, Seong MK. Predicting Radiation Resistance in Breast Cancer with Expression Status of Phosphorylated S6K1. Sci Rep. 2020 Jan 20;10(1):641. doi: 10.1038/s41598-020-57496-8. PMID: 31959810; PMCID: PMC6971275.

37. Ni J, Cheung BB, Beretov J, Duan W, Bucci J, Malouf D, Graham P, Li Y. CD44 variant 6 is associated with prostate cancer growth and chemo-/radiotherapy response in vivo. Exp Cell Res. 2020 Mar 15;388(2):111850. doi: 10.1016/j.yexcr.2020.111850. Epub 2020 Jan 16. PMID: 31954695.

38. Tanaka H, Kawaguchi M, Shoda S, Miyoshi T, Iwasaki R, Hyodo F, Mori T, Hara A, Tomita H, Matsuo M. Nuclear Accumulation of $\beta$-Catenin in Cancer Stem Cell Radioresistance and Stemness in Human Colon Cancer. Anticancer Res. 2019 Dec;39(12):6575-6583. doi: 10.21873/anticanres.13873. PMID: 31810923.

39. Elkashty OA, Abu Elghanam G, Su X, Liu Y, Chauvin PJ, Tran SD. Cancer stem cells enrichment with surface markers CD271 and CD44 in human head and neck squamous cell carcinomas.

Carcinogenesis. 2020 Jun 17;41(4):458-466. doi: 10.1093/carcin/bgz182. PMID: 31742606; PMCID: PMC7298622.

40. Klarmann GJ, Hurt EM, Mathews LA, Zhang X, Duhagon MA, Mistree T, Thomas SB, Farrar WL. Invasive prostate cancer cells are tumor initiating cells that have a stem cell-like genomic signature. Clin Exp Metastasis. 2009;26(5):433-46. doi:10.1007/s10585-009-9242-2. Epub 2009 Feb 17. PMID: 19221883; PMCID: PMC2782741.

41. Eke I, Cordes N. Focal adhesion signaling and therapy resistance in cancer[C]//Seminars in cancer biology. Academic Press. 2015;31:65-75.

42. Chikano Y, Domoto T, Furuta T, et al. Glycogen synthase kinase $3 \beta$ sustains invasion of glioblastoma via the focal adhesion kinase, Rac1, and c-Jun N-terminal kinase-mediated pathway[J]. Mol Cancer Ther. 2015;14(2):564-74.

43. Hehlgans S, Lange I, Eke I, et al. 3D cell cultures of human head and neck squamous cell carcinoma cells are radiosensitized by the focal adhesion kinase inhibitor TAE226[J]. Radiotherapy oncology. 2009;92(3):371-8.

44. Hou J, Zhou Z, Chen $X$, et al. HER2 reduces breast cancer radiosensitivity by activating focal adhesion kinase in vitro and in vivo[J]. Oncotarget. 2016;7(29):45186.

45. Alexi X, Berditchevski F, Odintsova E. The effect of cell-ECM adhesion on signalling via the ErbB family of growth factor receptors. Biochem Soc Trans. 2011 Apr;39(2):568 - 73. doi: 10.1042/BST0390568. PMID: 21428941.

\section{Figures}




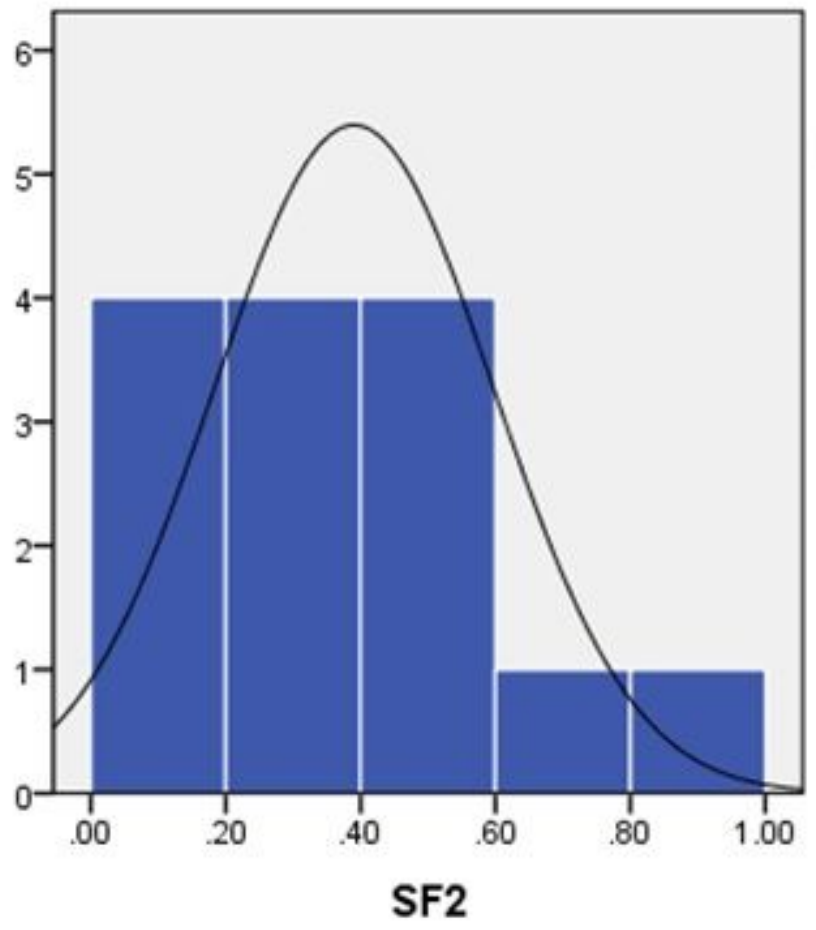

Figure 1

The mean SF2(surviving fraction at 2 Gy) distribution about fourteen lung adenocarcinoma.

Student's TTest $(P=\mathbf{0 . 8 2 2})$

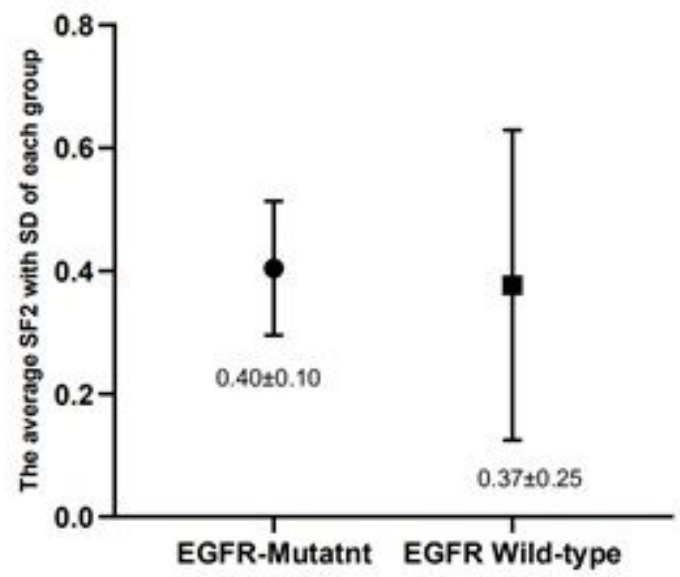

EGFR mutant status groups about fourteen lung adenocarcinoma cells

Figure 2

Radiosensitivity variations between EGFR mutant-type group and wild-type group. Abscissa is EGFR mutant status groups about fourteen lung adenocarcinoma cells and the ordinate is the average SF2 with SD of each group. Student' T-test was used to test the relationship between EGFR mutation and nonmutation and SF2. 
a.

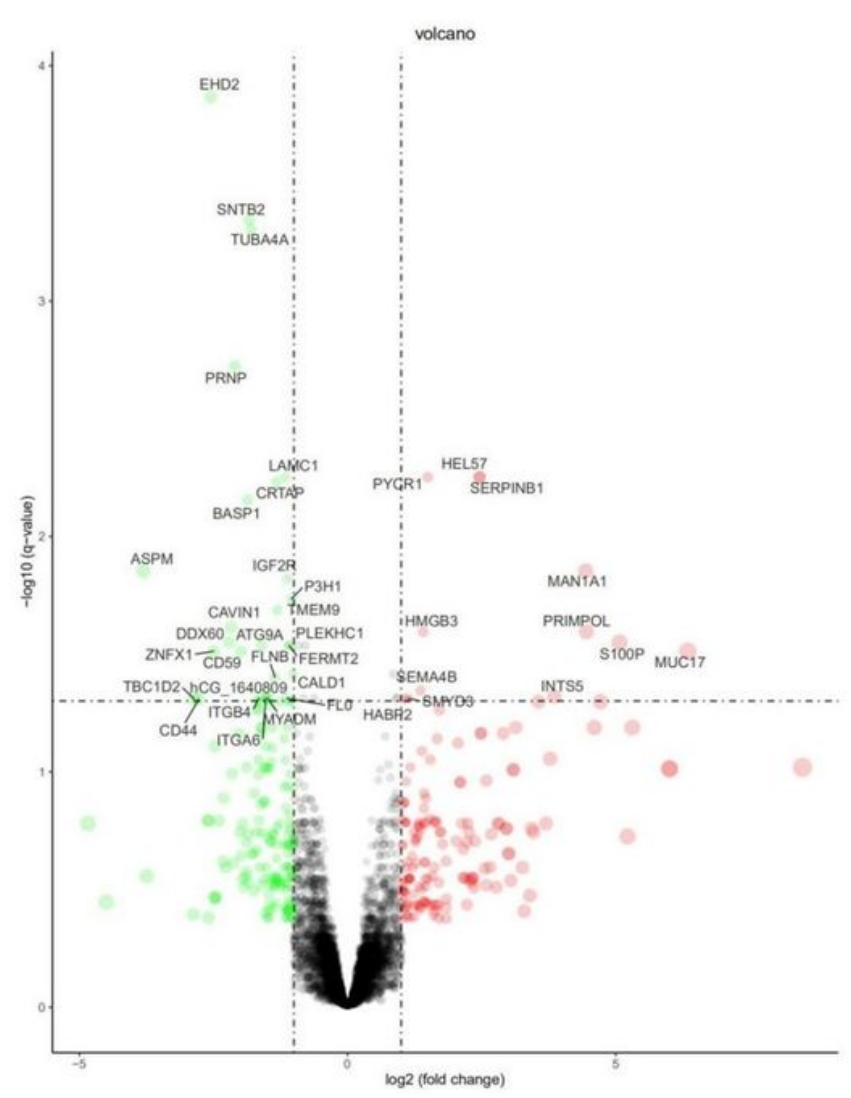

b

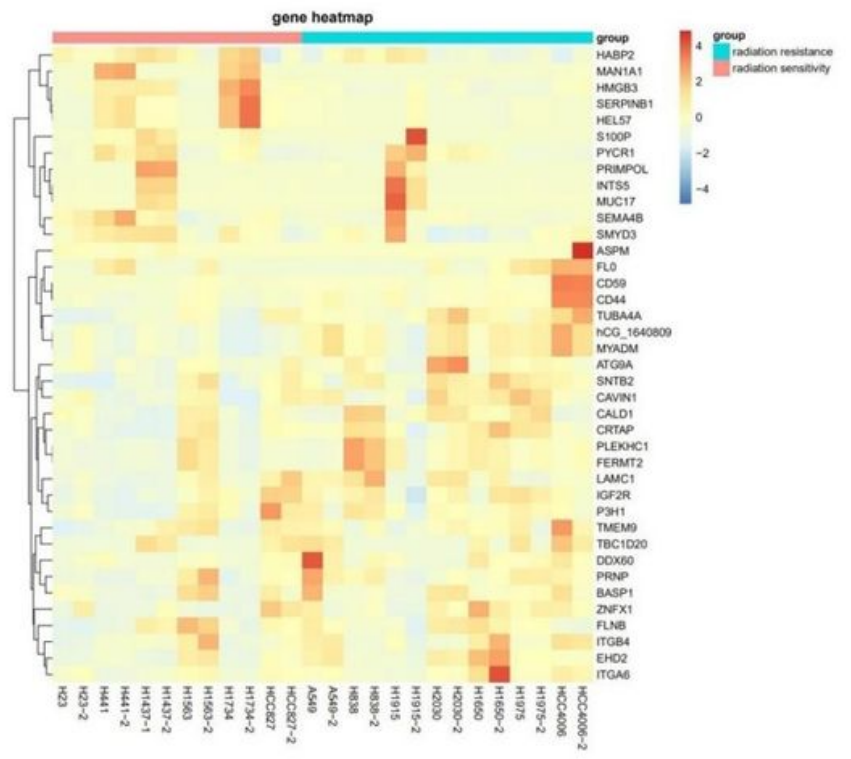

\section{Figure 3}

a.Volcano plot of DEPs(differently expressed proteins) Compared with radiation-resistant cells, the highly expressed proteins in radiation-sensitive cells were marked by red dots and the low-expressed proteins were marked with green dots. The horizontal line is foldchange $>1$, and the vertical line indicates P.adj < 0.05. Those who meet the conditions are considered to be differential proteins, and the gene name is marked. b, Heatmap of DEPs 

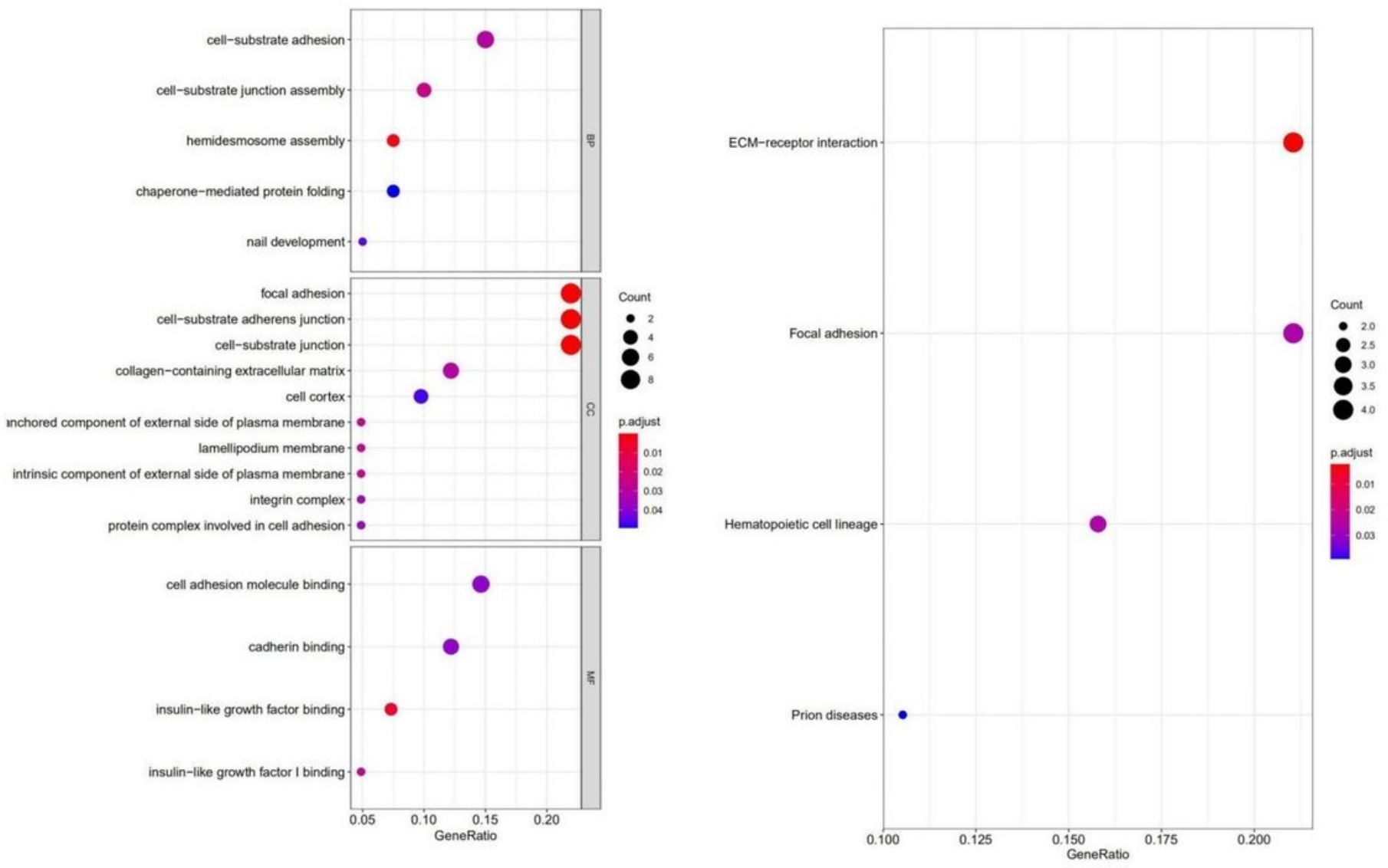

\section{Figure 4}

a. Dot plot of GO(Gene Ontology) GO including CC (cellular component), MF (molecular function), and BP (biological process). The terms assigned to the BP domain were cell-substrate adhesion and so on. The identified CC focal adhesion, cell-substrate adherents junction, cell-substrate junction, hemidesmosome. The identified MF terms were insulin-like growth factor binding, neuregulin binding. b.Dot plot of KEGG pathway The x-axis represents the gene ratio of DEPs(differently expressed proteins) throughout KEGG enrichments. The size of the circular dots indicates the number of enriched DEPs, with the intensity of circle color representing the-value of DEP,s differentially expressed protein. 
A

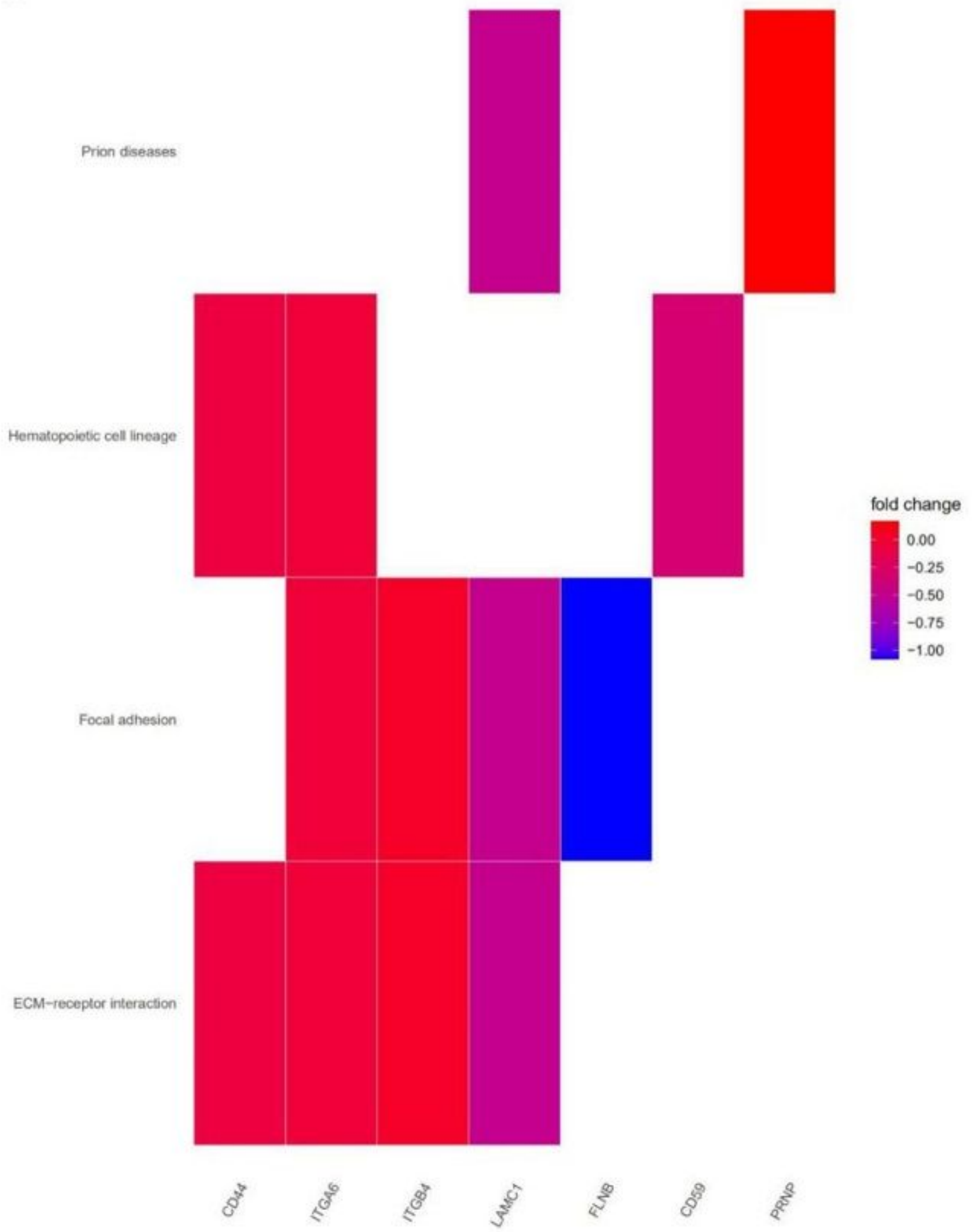

Figure 5

Heatmap plot of enriched terms. Heatmap-like functional classification figure display the relationships between DEPs(differently express proteins) and the KEGG pathway as a heatmap.LAMC1 involves ECMreceptor interaction, Focal adhesion, Prion diseases. ITGA6 involves ECM-receptor interaction, Focal adhesion, Hematopoietic cell lineage. ITGB4 involves ECM-receptor interaction, Focal adhesion. CD44 
involves ECM-receptor interaction, Prion diseases. It shows that these signal pathways can be connected by these molecules to form a network of signal pathways related to radiosensitivity.

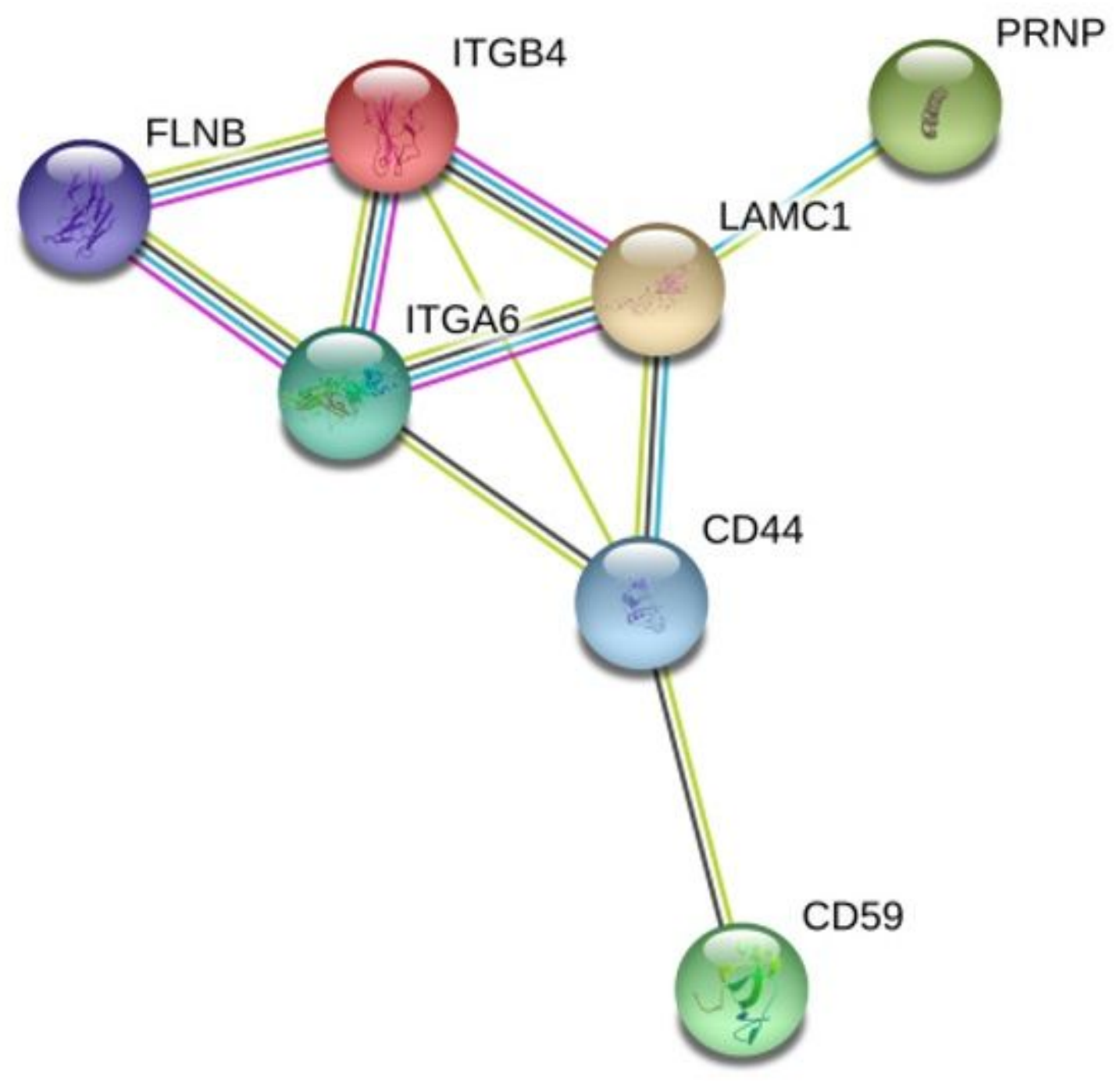

Figure 6

$\mathrm{PPI}($ protein and protein interaction) analysis The Protein-protein interaction(PPI) networks of all DEPs(differently express proteins) which used in KEGG enrichment.

\section{Supplementary Files}

This is a list of supplementary files associated with this preprint. Click to download.

- supplement.docx 